\title{
Viabilidad del uso de la radiación solar concentrada al proceso de sinterización de cobre
}

\author{
I. Cañadas", D. Martínez", J. Rodríguez"y J.M. Gallardo** \\ Resumen En la actualidad, el proceso de sinterizado, así como la práctica totalidad de los procesos que \\ se llevan a cabo en la industria, depende de las energías convencionales. Este trabajo \\ pretende demostrar la viabilidad del uso de una fuente de energía renovable como es la \\ Energía Solar Térmica en el sinterizado de cobre. Con objeto de realizar en el futuro una \\ evaluación de las fuerzas conductoras y de la trayectoria del material transportado en \\ función de las características del sistema y de la radicación incidente, se opta por diseñar \\ distintos dispositivos experimentales de empaquetamiento basados en modelos de \\ partículas ideales, en este caso cilindros, con los que se pretende simplificar la geometría del \\ sistema real basado en partículas compactadas, siendo evaluados experimentalmente en el \\ Horno Solar de la Plataforma Solar de Almería. Las muestras tratadas en atmósferas \\ ligeramente reductoras, desarrollan cuellos bien definidos y muestran una reducción de \\ porosidad, lo que permite demostrar la viabilidad del proceso.
}

Palabras clave Sinterizado. Cobre. Energía solar. Horno solar.

\section{Feasibility of concentrated solar energy utilization in copper sintering process}

\begin{abstract}
Sintering processes as well as all industrial processes depend on conventional energies. This work tries to demonstrate the feasibility of the application of a renewable power source, as Thermal Solar Energy, in copper sintering. In order to evaluate in the future driving forces and transported material path according to characteristics of the system and the incident radiation, different experimental devices based on ideal particle models, cylinders in this case, were designed to simplify the real system geometry based on compact powders. These devices were evaluated in the Plataforma Solar de Almería (PSA) Solar Furnace. Later analysis of the test pieces showed the feasibility of sintering copper in solar furnaces, the test pieces, treated in slightly reducing atmosphere, have developed well-defined necks and reduced porosity, which are typical characteristics of sintering.
\end{abstract}

Keywords Sintering. Copper. Solar energy. Solar furnace.

\section{INTRODUCCIÓN}

Las condiciones de trabajo y las elevadas temperaturas que se precisan en el sinterizado de cobre hacen que las instalaciones de Energía Solar Térmica que mejor se adaptan a las necesidades específicas del sinterizado sean los hornos solares. El diseño de estos hornos solares permite conseguir una concentración energética muy alta, pudiendo alcanzar valores por encima de $1.000 \mathrm{~W} / \mathrm{cm}^{2}$ aplicables de manera casi instantánea sobre la muestra a ensayar, y temperaturas de hasta $3.500{ }^{\circ} \mathrm{C}$, lo que los hace especialmente adecuados para el tratamiento de materiales.

\section{HORNOS SOLARES}

Constan esencialmente de un helióstato plano que realiza seguimiento solar continuo, un espejo parabólico concentrador, un atenuador de flujo y la zona de ensayos situada en el foco del concentra$\operatorname{dor}^{[1-3]}$ (Fig. 1).

El espejo captador plano -helióstato- refleja los rayos solares paralelos y horizontales sobre el disco

(*) CIEMAT - Plataforma Solar de Almería. P.O. Box 22, 04200 Tabernas, (Almería)

(**) Depart. de Ingeniería Mecánica y de los Materiales. Universidad de Sevilla. Av. del Descubrimiento s/n. 41092 Sevilla 


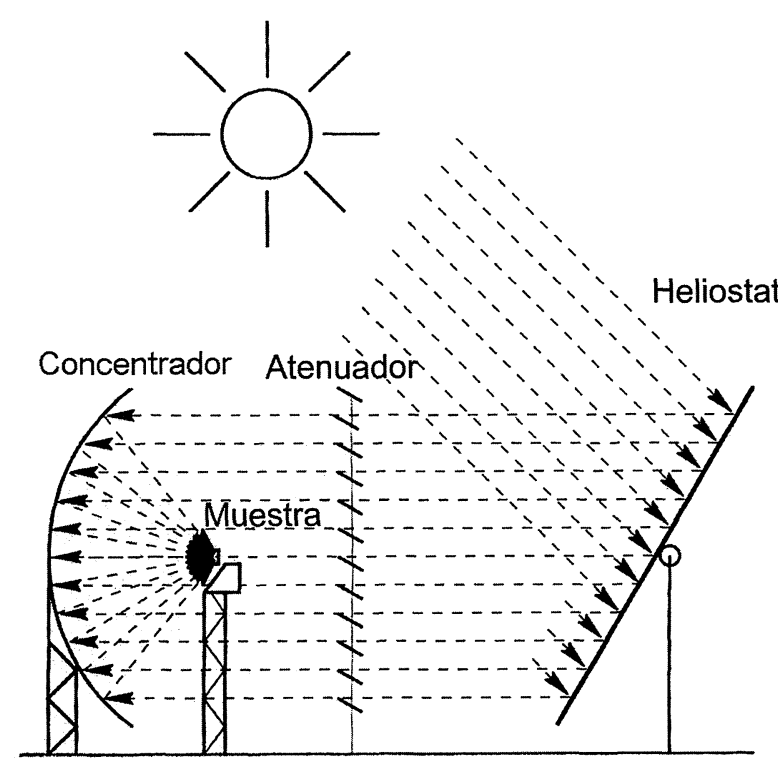

Figura 1. Esquema funcional del Horno Solar.

Figure 1. Schematic drawing of a Solar Furnace.

parabólico, el cual los vuelve a reflejar concentrándolos en su foco (área de ensayos). La cantidad de luz incidente se regula mediante el atenuador situado entre el concentrador y el helióstato. Bajo el foco se encuentra la mesa de ensayos que tiene movimiento en las tres dimensiones espaciales, permitiendo posicionar las probetas con gran exactitud en el foco.

\section{OBJETIVO}

Este trabajo pretende demostrar la viabilidad del uso de una fuente de energía renovable como es la Energía Solar Térmica en el sinterizado de cobre.

La geometría de polvos compactos es excesivamente compleja para el desarrollo futuro, de un modelo matemático que permita la precisa evaluación de las fuerzas conductoras y la trayectoria del material transportado. Esto conduce a optar por modelos de partículas ideales (esferas o cilindros) mediante los cuales se puede llegar a aproximar la geometría del sistema inicial y describir la cinética que rige los procesos de sinterización por mecanismos difusionales ${ }^{[4]}$.

Teniendo en cuenta las características del Horno y la densidad de flujo del foco se considera que un modelo de alambres (Fig. 2) es el más adecuado para su tratamiento en este tipo de hornos.

\section{PROCEDIMIENTO EXPERIMENTAL}

Para determinar la viabilidad del proceso de sinterizado en un Horno Solar se procederá a llevar a

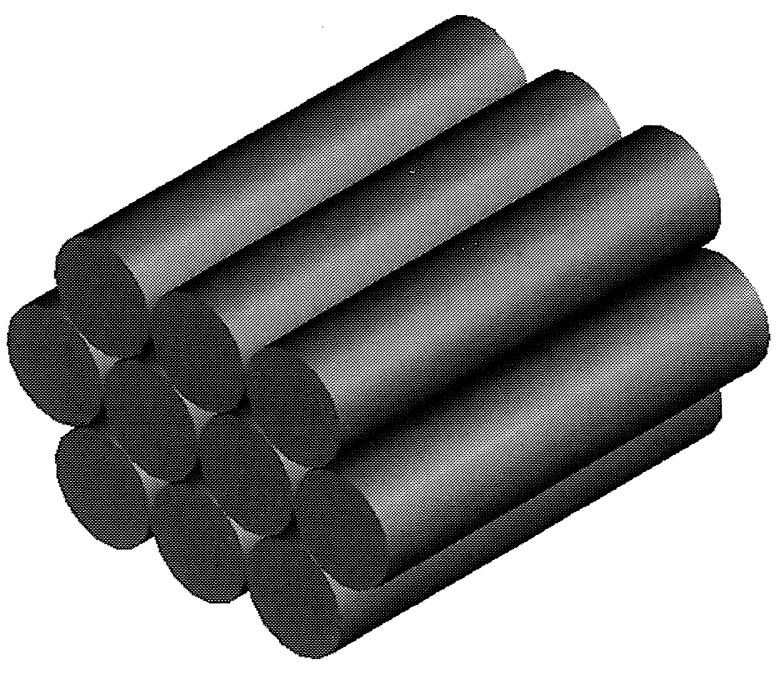

Figura 2. Modelo de alambres.

Figure 2. Wires model.

cabo la sinterización de una malla de alambres de cobre. El diseño de la probeta presenta dificultades debido a las características del Horno y de la radiación incidente. Se han desarrollado y evaluado dos dispositivos experimentales de empaquetamiento, obteniéndose diferentes resultados. En ambos dispositivos se ha empleado cobre electrolítico recocido, de bajo contenido en oxígeno $(<200$ ppm).

\subsection{Dispositivo 1. Alambres bobinados}

Se realiza un diseño de múltiples capas de alambre de cobre bobinado sobre un carrete también de cobre, similar al desarrollado por Alexander y Balluffi ${ }^{[5]}$. En nuestro caso, la distribución de los alambres sobre el soporte es la que se muestra en la figura 3, siendo el diámetro de los alambres de 0,1 mm.

La probeta se coloca en el interior de una cámara de vacío especialmente diseñada para su uso en el Horno Solar. Esta cámara está dotada con un sistema de preparación de gases, lo que nos permite trabajar en atmósfera controlada; en este caso se proporciona una atmósfera ligeramente reductora mediante el gas HYD45, $5 \% \mathrm{H}_{2}-95 \% \mathrm{~N}_{2}$, para evitar la oxidación del cobre.

La radiación solar concentrada se aplica directamente sobre la superficie de la muestra a ensayar a través de la ventana de cuarzo situada en la parte frontal de la cámara, tal y como se indica en la figura 4.

\subsection{Dispositivo 2. Alambres enderezados}

Se elabora un soporte de cobre que contendrá los alambres previamente enderezados. En este caso se 


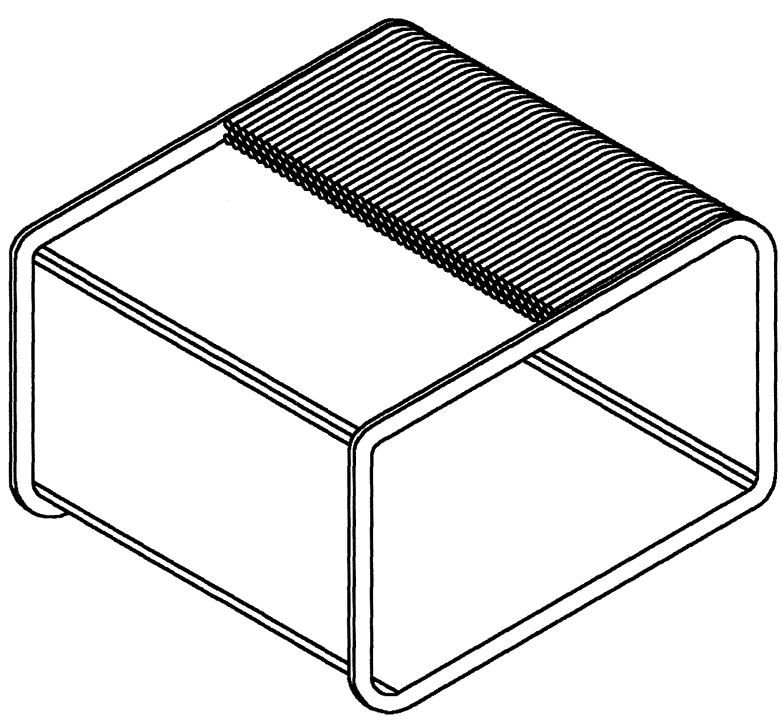

Figura 3. Diseño de alambres bobinados.

Figure 3. Wires winding design.

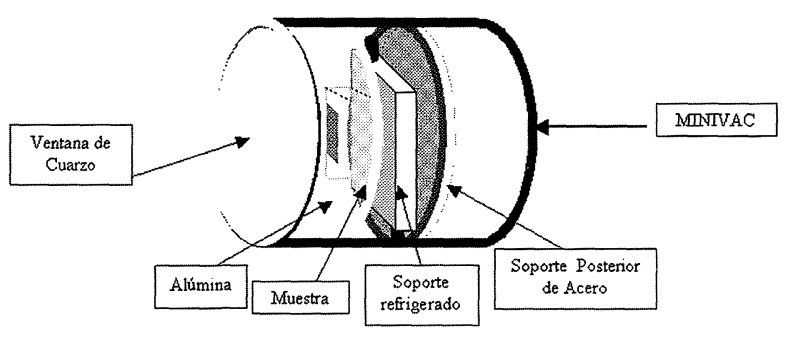

Figura 4. Dispositivo de alambres bobinados en el interior de la cámara.

Figure 4. Wires winding specimen inside of the chamber.

emplearán alambres de $2 \mathrm{~mm}$ de diámetro. El contacto inicial entre los alambres de cobre se asegura manteniendo durante los ensayos unas sujeciones diseñadas de manera que éstas ejerzan la presión mínima suficiente para favorecer el contacto entre alambres adyacentes (Fig. 5).

Para la realización de estos ensayos se utilizará la cámara en posición horizontal, haciendo uso de un espejo de redireccionamiento que modifique $90^{\circ}$ la dirección de la radiación incidente.

Una vez colocada la pieza sobre un soporte de acero se protege con una chapa de alúmina en la que se ha realizado un orificio con las dimensiones de la superficie a tratar. La alúmina protege los accesorios necesarios para el ensayo, mientras que el orificio practicado en ella permite controlar la zona de exposición a la radiación incidente. La disposición de la probeta en la cámara es la que se muestra en la figura 6 .

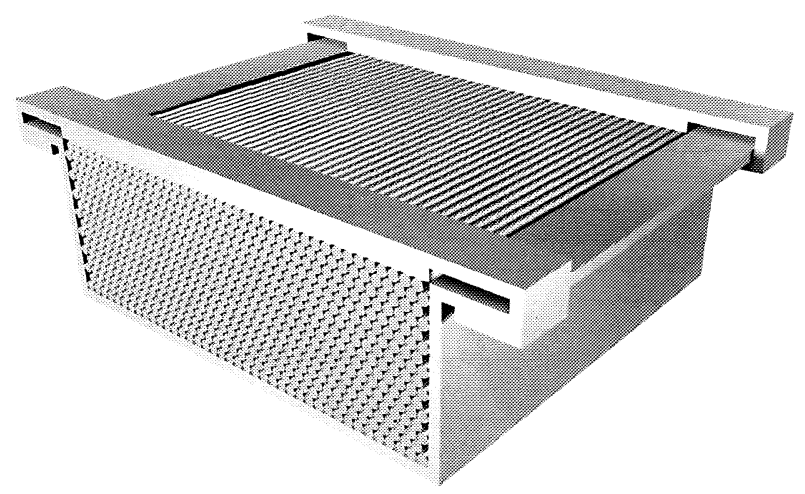

Figura 5. Diseño de alambres enderezados.

Figure 5. Wires straightened design.

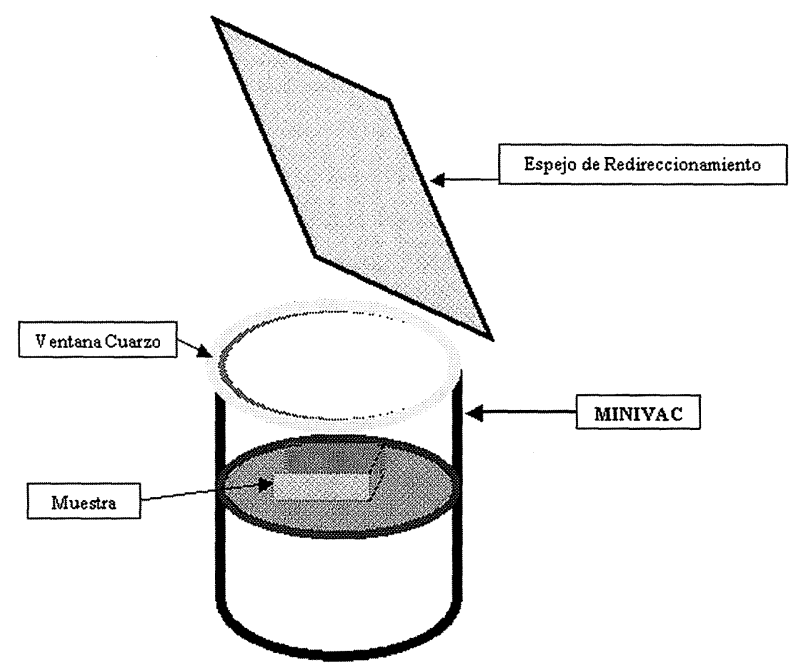

Figura 6. Dispositivo de alambres enderezados en el interior de la cámara.

Figure 6. Wire Straightened design specimen inside of the chamber.

Se trabaja en atmósfera ligeramente reductora, pero para evitar posibles problemas derivados de la presencia de pequeñas películas de óxido en la superficie de los alambres, éstos son tratados previamente con alcohol isopropílico en ultrasonidos.

\section{RESULTADOS}

Por las características de la radiación incidente se determina que una probeta de alambres bobinados similar a la que hasta ahora ha sido empleada para el estudio de los mecanismos que tienen lugar durante el sinterizado ${ }^{[5-8]}$ no es adecuada para su tratamiento en el Horno Solar ya que al aplicar un tratamiento térmico con energía solar concentrada sobre la superficie de este tipo de muestras, las capas más externas se dilatan, quedando aisladas del 
resto de la bobina, lo que provoca la formación de discontinuidades en la probeta que llevan asociadas una notable disminución en la cantidad de calor que se transmite a las capas interiores del bobinado, ya que en estas discontinuidades la transmisión de calor se realiza por convección y no por conducción, dando lugar a zonas de tratamiento claramente diferenciadas dentro de la probeta.

Para el segundo dispositivo, la probeta con alambres enderezados, el soporte presenta únicamente dos superficies laterales con el fin de no crear tensiones que impidan la dilatación longitudinal libre de los alambres de cobre que se producía con el bobinado de alambre. En este caso, el ensayo se realiza de manera satisfactoria.

El nuevo diseño responde a las expectativas, ya que no se han producido tensiones debidas a la dilatación de los alambres por la temperatura y no parece presentar problemas para que, en condiciones adecuadas, se produzca su sinterización.

Se han realizado ensayos a temperaturas que oscilan entre 850 y $1.050^{\circ} \mathrm{C}$ y tiempos de exposición variables. En líneas generales, se puede decir que, debido a la elevada conductividad térmica del cobre, las probetas tratadas mediante radiación solar térmica concentrada incidente sobre su superficie superior se muestran homogéneas en todo su volumen, no encontrándose diferencias apreciables entre las diferentes capas.

De manera general podemos decir que se observa que para temperaturas inferiores a $1.000^{\circ} \mathrm{C}$ y tiempos entre 2 y $180 \mathrm{~min}$, tiene lugar la formación de cuellos en un número reducido de contactos, dependiendo la longitud de los cuellos formados de la duración del tratamiento. Así, para un tratamiento de $60 \mathrm{~min}$ a $1.000{ }^{\circ} \mathrm{C}$ la longitud media de los cuellos formados entre alambres adyacentes es de $88,15 \mu \mathrm{m}$.

Sin embargo, en los ensayos realizados a 1.050 ${ }^{\circ} \mathrm{C}$ se observa que la formación de cuellos se produce siempre que existe un contacto inicial; así, para un tratamiento de $2 \mathrm{~min}$ a $1.050^{\circ} \mathrm{C}$ la longitud media de los cuellos formados es de 186,04 $\mu \mathrm{m}$, pudiéndose observar las siguientes micrografías (Fig. 7).

\section{DISCUSIÓN}

Macroscópicamente, se puede observar que los resultados obtenidos en el Horno Solar son similares a los obtenidos en investigaciones similares realizadas anteriormente en hornos convencionales ya que, de manera general, podemos decir que a tem-
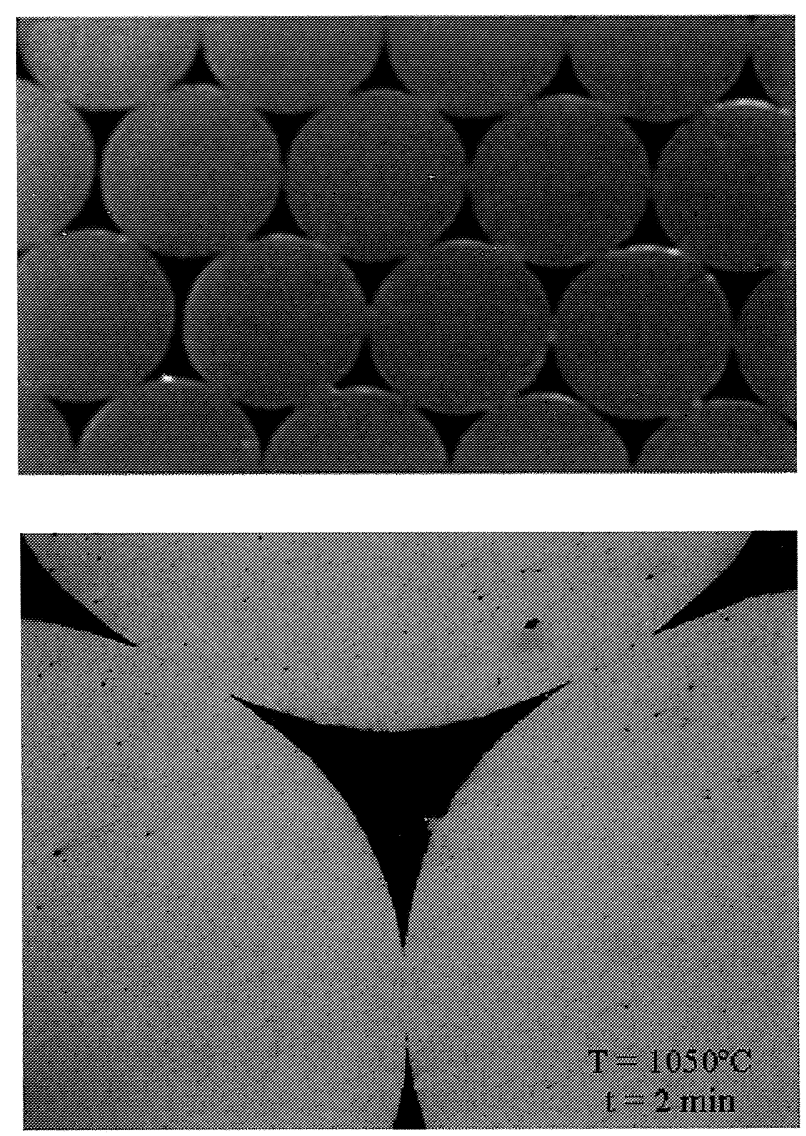

Figura 7. Sección de probeta de alambres tras su sinterizado a $1050^{\circ} \mathrm{C}$ durante $2 \mathrm{~min}$. en Horno Solar.

Figure 7. Cross-section of wires specimen after sintering at $1050^{\circ} \mathrm{C}$ for 2 minutes in a Solar Furnace.

peraturas inferiores a $1.050{ }^{\circ} \mathrm{C}$ el porcentaje de cuellos formados entre alambres adyacentes es bajo, mientras que a partir de esa temperatura la formación de cuellos es elevada, observándose una mayor homogeneidad en las muestras.

Así, en los experimentos realizados por Alexander y Balluffi sobre un diseño de múltiples capas de alambre bobinado sobre un carrete de cobre, en un primer caso, los alambres de cobre, de diámetro $0,128 \mathrm{~mm}$, fueron sometidos a tratamientos térmicos a $1.075^{\circ} \mathrm{C}$ y tiempos superiores a las 4 h. Los resultados y micrografías que obtuvieron muestran que a esa temperatura se produce la formación de cuellos desde los primeros resultados analizados.

Realizaron otra serie de ensayos a temperatura ligeramente inferior, $1.000{ }^{\circ} \mathrm{C}$, y estudiaron los resultados para tiempos superiores a las $8 \mathrm{~h}$. Observaron que para tiempos de $8 \mathrm{~h}$ prácticamente no se forman cuellos; a tiempos superiores observaron una mayor homogeneidad en la pieza y un mayor número de cuellos. 
Como se ha indicado, en ambos casos, los resultados son similares a los que se obtienen en el Horno Solar.

\section{CONCLUSIONES}

- El análisis preliminar de las piezas ensayadas muestra la viabilidad del sinterizado de cobre en hornos solares, desarrollando las probetas tratadas cuellos bien definidos y reduciendo su porosidad, características propias del sinterizado.

- La evaluación de dos dispositivos experimentales para el sinterizado de un modelo cilíndrico en el Horno Solar arroja resultados satisfactorios para un diseño de alambres de cobre enderezados, no siendo experimentalmente adecuado el uso de un bobinado de alambre de cobre, empleado por otros autores en el caso de hornos convencionales.

- Actualmente se está estudiando la mejora del proceso que permita tratar superficies mayores con una mayor uniformidad térmica.

\section{Agradecimientos}

Proyecto financiado por el Ministerio de Ciencia y Tecnología dentro del Programa Nacional de In- vestigación Científica, Desarrollo e Innovación Tecnológica, Referencia. REN2003-09247-C04-01

\section{REFERENCIAS}

[1] D. MartíneZ y J. RodríGuez, Rev. Metal. Madrid 34 (1998) 104-108.

[2] D. Martínez y J. Rodríguez, Surface Modification Technologies XI, Institute of Materials Pub., Londres, Inglaterra, 1998, pp. 441-447.

[3] I. Cañadas, D. Martínez, J. Rodríguez. Bol. Soc. Esp. Ceram. y Vidrio 43 (2004) 591-595.

[4] J. Svoboda, H. Riedel, Acta Metall. Mater. 43 (1995) 599. 606.

[5] B.H. AleXander, R.W. Balluffi, Acta Mater. (1957) 666677.

[6] F.V. Lenel, Powder Metallurgy Principles and Applications, Metal Powder Industries Federation, Princeton, New Jersey, Estados Unidos, 1980, pp. 229-239.

[7] W.D. Jones, Fundamental Principles of Powder Metallurgy, Edward Arnold Publishers, Londres, Reino Unido, 1960, $386-420$

[8] H.H. Hausner, W.E. Smith, Modern Developments in Powder Metallurgy, Metal Powder Industries Federation, Vol 6, Princeton, New Jersey, Estados Unidos, 1973, 215-235. 